\title{
Impact of a Multicenter, Mentored Quality Collaborative on Hospital-Associated Venous Thromboembolism
}

\author{
lan Jenkins, MD, SFHM ${ }^{1 *}$, Tamra O'Bryan, MHA, RHIA, CPHQ ${ }^{2}$, \\ Janet Holdych, PharmD, CPHQ², Gregory Maynard, MD, MS, $\mathrm{MHM}^{3}$
}

${ }^{1}$ Department of Medicine, University of California San Diego Health System, San Diego, California; ${ }^{2}$ Dignity Health, San Francisco, California; ${ }^{3}$ University of California Davis Medical Center, Sacramento, California.

BACKGROUND: Reliable prophylaxis of hospitalassociated venous thromboembolism (HA-VTE) is not achieved in many hospitals. Efforts to improve prophylaxis have had uneven results.

OBJECTIVE: To reduce HA-VTE with a scalable quality improvement collaborative.

DESIGN: A prospective, unblinded, open-intervention study with historical controls.

PARTICIPANTS AND SETTING: All adult inpatients at 35 community hospitals in California, Arizona, and Nevada.

INTERVENTIONS: A centrally supported collaborative implementing standardized VTE risk assessment and prophylaxis. Protocols were developed with 9 "pilot" sites, which received individualized mentoring. Finished protocols were disseminated to 26 "spread" sites, which received improvement webinars without mentoring. Active surveillance for real-time correction of suboptimal prophylaxis was funded in pilot sites and encouraged in spread sites. Planning and minimal improvement work began in 2011; most implementation occurred in 2012 and 2013.
MEASUREMENTS: Rates of per-protocol prophylaxis (at pilot sites), and compliance with The Joint Commission VTE measures (all sites), were monitored starting in January 2012. The International Classification of Diseases, 9th Edition-Clinical Modification codes were used to determine the rates of HA-VTE within 30 days of discharge, heparininduced thrombocytopenia, and anticoagulation adverse events; preimplementation (2011) rates were compared with postimplementation (2014) rates.

RESULTS: Protocol-appropriate prophylaxis rates and The Joint Commission measure compliance both reached $97 \%$ in 2014, up from 70\% to $89 \%$ in 2012 and 2013. Five thousand three hundred and seventy HA-VTEs occurred during 1.16 million admissions. Four hundred twenty-eight fewer HA-VTEs occurred in 2014 than in 2011 (relative risk $0.78 ; 95 \%$ confidence interval, $0.73-0.85)$. HA-VTEs fell more in pilot sites than spread sites $(26 \%$ vs $20 \%)$. The rates of adverse events were reduced or unchanged.

CONCLUSIONS: Collaborative efforts were associated with improved prophylaxis rates and fewer HA-VTEs. Journal of Hospital Medicine 2018;13:462-469. Published online first February 13, 2018. (C) 2018 Society of Hospital Medicine

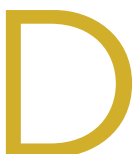

eep venous thrombosis and pulmonary embolism collectively known as venous thromboembolism (VTE), affect up to 600,000 Americans a year. ${ }^{1}$ Most of these are hospital-associated venous thromboembolisms (HA-VTE). ${ }^{1}{ }^{2}$ VTE poses a substantial risk of mortality and long-term morbidity, and its treatment poses a risk of major bleeding. ${ }^{1}$ As appropriate VTE prophylaxis ("prophylaxis") can reduce the risk of VTE by $40 \%$ to $80 \%$ depending on the patient population, ${ }^{3} \mathrm{VTE}$ risk assessment and prophylaxis is en-

\footnotetext{
*Address for correspondence: lan Jenkins, MD, SFHM, Clinical Professor of Medicine, Department of Medicine, University of California San Diego Health System,200 W Arbor Drive, MC 8485, San Diego, CA 92103; Telephone: 619884-0334; Fax: 619-543-8255; E-mail: ihjenkins@ucsd.edu

Additional Supporting Information may be found in the online version of this article.
}

Received: August 4, 2017; Revised: November 17, 2017;

Accepted: December 5, 2017

๑ 2018 Society of Hospital Medicine DOI 10.12788/jhm.2942 dorsed by multiple guidelines ${ }^{4.7}$ and supported by regulatory agencies. . $^{8-10}$

However, despite extensive study, consensus about the impact of prophylaxis ${ }^{4,11}$ and the optimal method of risk assessment $4,5,7,12$ is lacking. Meanwhile, implementation of prophylaxis in real-world settings is poor; only $40 \%$ to $60 \%$ of at-risk patients receive prophylaxis, ${ }^{13}$ and as few as $<20 \%$ receive optimal prophylaxis. ${ }^{14}$ Both systematic reviews ${ }^{15,16}$ and experience with VTE prevention collaboratives ${ }^{17,18}$ found that multifaceted interventions and alerts may be most effective in improving prophylaxis rates, but without proof of improved VTE rates. ${ }^{15}$ There is limited experience with large-scale VTE prevention. Organizations like The Joint Commission (TJC) ${ }^{8}$ and the Surgical Care Improvement Project have promoted quality measures but without clear evidence of improvement. ${ }^{19}$ In addition, an analysis of over 20,000 medical patients at 35 hospitals found no difference in VTE rates between high- and low-performing hospitals, ${ }^{20}$ suggesting that aggressive prophylaxis efforts may not reduce VTE, at least among medical patients. ${ }^{21}$ However, a 5-hospital University of California collaborative was associated 


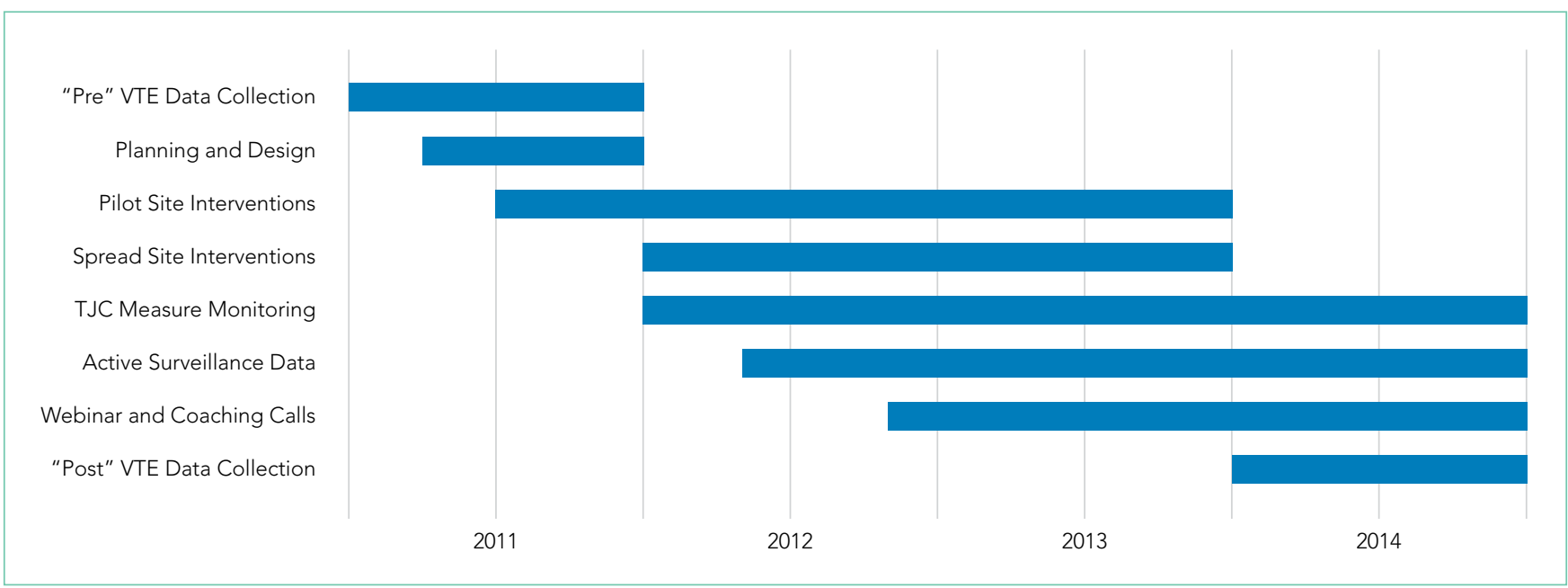

FIG 1. Gantt chart showing the timeframe of interventions across the 4-year study period. TJC measure monitoring: monitoring of The Joint Commission VTE-1 and 2 metric compliances. Active surveillance data: monitoring of protocol compliant prophylaxis rates. Webinar and coaching calls continued after the study timeframe.

with improved VTE rates, chiefly among surgical patients. ${ }^{22}$

In 2011, Dignity Health targeted VTE for improvement after investigations of potentially preventable HA-VTE revealed variable patterns of prophylaxis. In addition, improvement seemed feasible because there is a proven framework for VTE quality improvement (Ql) projects ${ }^{17,18}$ and a record of success with the following 3 specific strategies: quality mentorship, ${ }^{23}$ use of a simple VTE risk assessment method, and active surveillance (real-time monitoring targeting suboptimal prophylaxis with concurrent intervention). This active surveillance technique has been used successfully in prior improvement efforts, often termed measure-vention. ${ }^{17,18,22,24}$

\section{METHODS}

\section{Setting and Participants}

The QI collaborative was performed at 35 Dignity Health community hospitals in California, Arizona, and Nevada. Facilities ranged from 25 to 571 beds in size with a mixture of teaching and nonteaching hospitals. Prior to the initiative, prophylaxis improvement efforts were incomplete and inconsistent at study facilities. All adult acute care inpatients at all facilities were included except rehabilitation, behavioral health, skilled nursing, hospice, other nonacute care, and inpatient deliveries.

\section{Design Overview}

We performed a prospective, unblinded, open-intervention study of a $\mathrm{Ql}$ collaborative in 35 community hospitals and studied the effect on prophylaxis and VTE rates with historical controls. The 35 hospitals were organized into 2 cohorts. In the "pilot" cohort, 9 hospitals (chosen to be representative of the various settings, size, and teaching status within the Dignity system) received funding from the Gordon and Betty Moore Foundation (GBMF) for intensive, individualized Ql mentorship from experts as well as active surveillance (see "Interventions"). The pilot sites led the development of the VTE risk assessment and prophylaxis protocol ("VTE protocol"), measures, order sets, implementation tactics, and lessons learned, assisted by the mentor experts. Dissemination to the 26-hospital "spread" cohort was facilitated by the Dignity Health Hospital Engagement Network (HEN) infrastructure.

\section{Timeline}

Two of the pilot sites, acting as leads on the development of protocol and order set tools, formed improvement teams in March 2011, 6 to 12 months earlier than other Dignity sites. Planning and design work occurred from March 2011 to September 2012. Most implementation at the 35 hospitals occurred in a staggered fashion during calendar year (CY) 2012 and 2013 (see Figure 1). As few changes were made until mid2012, we considered CY 2011 the baseline for comparison, CY 2012 to 2013 the implementation years, and CY 2014 the postimplementation period.

The project was reviewed by the Institutional Review Board (IRB) of Dignity Health and determined to be an IRB-exempt Ql project.

\section{Interventions}

\section{Collaborative Infrastructure}

Data management, order set design, and hosted webinar support were provided centrally. The Dignity Health Project Lead (T.O.) facilitated monthly web conferences for all sites beginning in November 2012 and continuing past the study period (Figure 1), fostering a monthly sharing of barriers, solutions, progress, and best practices. These calls allowed for data review and targeted corrective actions. The Project Lead visited each hospital to validate that the recommended practices were in place and working.

\section{Multidisciplinary Teams}

Improvement teams formed between March 2011 and September 2012. Members included a physician champion, frontline nurses and physicians, an administrative liaison, pharmacists, quality and data specialists, clinical informatics staff, and stakeholders from key clinical services. Teams met at least monthly at each site. 


\section{Physician Mentors}

The 9 pilot sites received individualized mentorship provided by outside experts (IJ or GM) based on a model pioneered by the Society of Hospital Medicine's (SHM) Mentored Implementation programs. ${ }^{23}$ Each pilot site completed a self-assessment survey ${ }^{17}$ (see supplementary Appendix A) about past efforts, team composition, current performance, aims, barriers, and opportunities. The mentors reviewed the completed questionnaire with each hospital and provided advice on the VTE protocol and order set design, measurement, and benchmarking during 3 webinar meetings scheduled at 0, 3, and 9 months, plus as-needed e-mail and phone correspondence. After each webinar, the mentors provided detailed improvement suggestions (see supplementary Appendix B). Several hospitals received mentor site visits, which focused on unit rounding, active surveillance, staff and provider education, and problem-solving sessions with senior leadership, physician leadership, and the improvement team.

\section{VTE Protocol}

After a literature review and consultation with the mentors, Dignity Health developed and implemented a VTE protocol, modified from a model used in previous improvement efforts. ${ }^{18,22-24}$ Its risk assessment method is often referred to as a "3 bucket" model because it assigns patients to high-, moderate-, or low-risk categories based on clinical factors (eg, major orthopedic surgery, prior VTE, and others), and the VTE protocol recommends interventions based on the risk category (see supplementary Appendix C). Dignity Health was transitioning to a single electronic health record (Cerner Corporation, North Kansas City, MO) during the study, and study hospitals were using multiple platforms, necessitating the development of both paper and electronic versions of the VTE protocol. The electronic version required completion of the VTE protocol for all inpatient admissions and transfers. The VTE protocol was completed in November 2011 and disseminated to other sites in a staggered fashion through November 2012. Completed protocols and improvement tips were shared by the project lead and by webinar sessions. Sites were also encouraged to implement a standardized practice that allowed nurses to apply sequential compression devices to at-risk patients without physician orders when indicated by protocol, when contraindications such as vascular disease or ulceration were absent.

\section{Education}

Staff were educated about the VTE protocol by local teams, starting between late 2011 and September 2012. The audience (physicians, nurses, pharmacists, etc.) and methods (conferences, fliers, etc.) were determined by local teams, following guidance by mentors and webinar content. Active surveillance provided opportunities for in-the-moment, patient-specific education and protocol reinforcement. Both mentors delivered educational presentations at pilot sites.

\section{Active Surveillance}

Sites were encouraged to perform daily review of prophylaxis adequacy for inpatients and correct lapses in real time (both under- and overprophylaxis). Inappropriate prophylaxis orders were addressed by contacting providers to change the order or document the rationale not to. Lapses in adherence to prophylaxis were addressed by nursing correction and education of involved staff. Active surveillance was funded for 10 hours a week at pilot sites. Spread sites received only minimal support from HEN monies. All sites used daily prophylaxis reports, enhanced to include contraindications like thrombocytopenia and coagulopathy, to facilitate efforts. Active surveillance began in May 2012 in the lead pilot hospitals and was implemented in other sites between October 2012 and February 2013.

\section{Metrics \\ Prophylaxis Rates}

Measurement of prophylaxis did not begin until 2012 to 2013; thus, the true baseline rate for prophylaxis was not captured. TJC metrics (VTE-1 and VTE-2) ${ }^{25}$ were consolidated into a composite TJC prophylaxis rate from January 2012 to December 2014 for both pilot and spread hospitals. These measures assess the percentage of adult inpatients who received VTE prophylaxis or have documentation of why no prophylaxis was given the day of or day after hospital admission (VTE-1) or the day of or day after ICU admission or transfer (VTE-2). These measures are met if any mechanical or pharmacologic prophylaxis was delivered.

In addition to the TJC metric, the 9 pilot hospitals monitored rates of protocol-compliant prophylaxis for 12 to 20 months. Each patient's prophylaxis was considered protocol compliant if it was consistent with the prophylaxis protocol at the time of the audit or if contraindications were documented (eg, patients eligible for, but with contraindications to, pharmacologic prophylaxis had to have an order for mechanical prophylaxis or documented contraindication to both modalities). As this measure was initiated in a staggered fashion, the rate of protocol-compliant prophylaxis is summarized for consecutive months of measurement rather than consecutive calendar months.

\section{HA-VTE Rates}

VTE events were captured by review of electronic coding data for the International Classification of Diseases, 9th Revision (ICD-9) codes 415.11-415.19, 453.2, 453.40-453.42, and 453.8453.89. HA-VTE was defined as either new VTE not present on admission (NPOA HA-VTE) or new VTE presenting in a readmitted patient within 30 days of discharge (Readmit HA-VTE). Cases were stratified based on whether the patient had undergone a major operation (surgery patients) or not (medical patients) as identified by Medicare Services diagnosis-related group codes.

\section{Control Measures}

Potential adverse events were captured by review of electronic coding data for ICD-9 codes 289.84 (heparin-induced thrombocytopenia [HIT]) and E934.2 (adverse effects because of anticoagulants). 


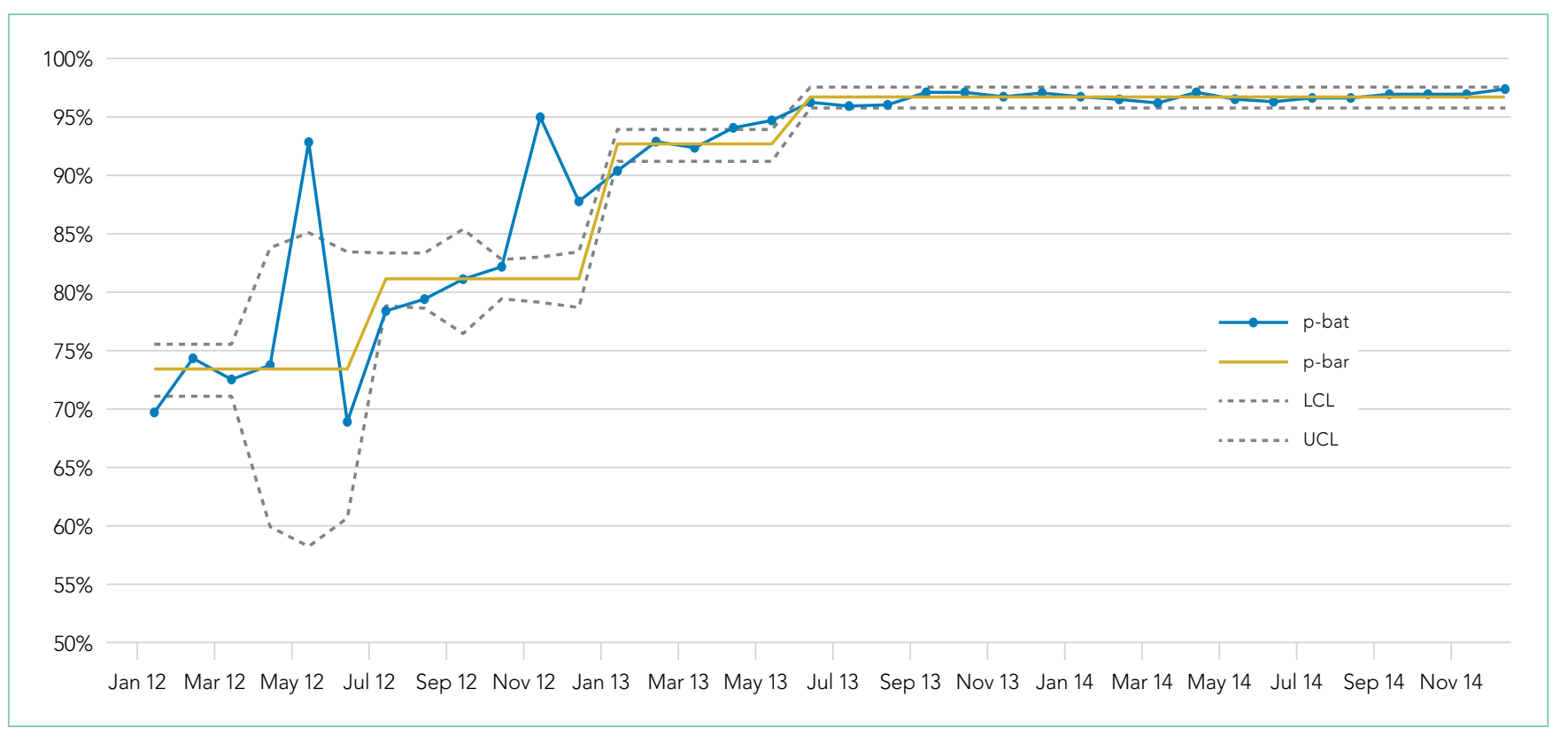

FIG 2. The Joint Commission (TJC) composite VTE-1 and VTE-2 rates of VTE prophylaxis compliance in the 35-site cohort (mean 1397 observations per month). Measured proportion in the sample, p-hat; average proportion in the sampled time-frame, p-bar. Abbreviations: LCL, lower confidence limit; UCL, upper confidence limit.

\section{Statistical Analysis}

Statistical process control charts were used to depict changes in prophylaxis rates over the 3 years for which data was collected. For VTE and safety outcomes, Pearson $\chi^{2}$ value with relative risk (RR) calculations and $95 \%$ confidence intervals (Cls) were used to compare proportions between groups at baseline (CY 2011) versus postimplementation (CY 2014). Differences between the means of normally distributed data were calculated, and a $95 \% \mathrm{Cl}$ for the difference between the means was performed to assess statistical difference. Nonparametric characteristics were described by quartiles and interquartile range, and the 2-sided Mann-Whitney $U$ test was performed to assess statistical difference between the CY 2011 and CY 2014 period.

\section{Role of the Funding Source}

The GBMF funded the collaborative and supported authorship of the manuscript but had no role in the design or conduct of the intervention, the collection or analysis of data, or the drafting of the manuscript.

\section{RESULTS}

\section{Population Demographics}

There were 1,155,069 adult inpatient admissions during the 4-year study period $(264,280$ in the 9 pilot sites, 890,789 in the 26 spread sites). There were no clinically relevant changes in gender distribution, mortality rate, median age, case mix index, or hospital length of stay in 2011 versus 2014. Men comprised $47.1 \%$ of the patient population in 2011 and $47.7 \%$ in 2014. The mortality rate was $2.7 \%$ in both years. Median age was 62 in 2011 and 63 in 2014. The mean case mix index (1.58 vs 1.65) and mean length of stay (4.29 vs 4.33 days) were similar in the 2 time periods.

\section{Prophylaxis Rates \\ TJC Prophylaxis rates}

There were 46,418 observations of TJC prophylaxis rates between January 2012 and December 2014 (mean of 1397 observations per month) in the cohort. Early variability gave way to consistent performance and tightened control limits, coinciding with widespread implementation and increased number of audits. TJC prophylaxis rates climbed from $72.2 \%$ in the first quarter of 2012 to $95 \%$ by May 2013. TJC prophylaxis rates remained $>95 \%$ thereafter, improving to $96.8 \%$ in 2014 (Pearson $\chi^{2} P<.001$ ) (Figure 2).

\section{Rates of Protocol-Compliant Prophylaxis}

There were 34,071 active surveillance audits across the 20 months of reporting in the pilot cohort (mean, 1817 audits per month). The rate of protocol-compliant prophylaxis improved from $89 \%$ at month 1 of observation to $93 \%$ during month 2 and $97 \%$ by the last 3 months (Pearson $\chi^{2} P<.001$ for both comparisons).

\section{HA-VTE \\ HA-VTE characteristics}

Five thousand three hundred and seventy HA-VTEs occurred during the study. The HA-VTE rate was higher in surgical patients $(7.4 / 1000)$ than medical patients (4.2/1000) throughout the study (Figure 3 ). Because only $32.8 \%$ of patients were surgical, however, $51 \%$ (2740) of HA-VTEs occurred in medical patients and $49 \%$ occurred (2630) in surgical patients. In medical patients, most HA-VTEs occurred postdischarge (2065 of 2740; $75 \%)$; in surgical patients, most occurred during the index admission (1611 of $2630 ; 61 \%$ ).

\section{Improved HA-VTE over Time}

Four hundred twenty-eight fewer HA-VTEs occurred in 2014 than in 2011 (RR 0.78; 95\% Cl, 0.73-0.85) (Table and Figure 3). Re- 


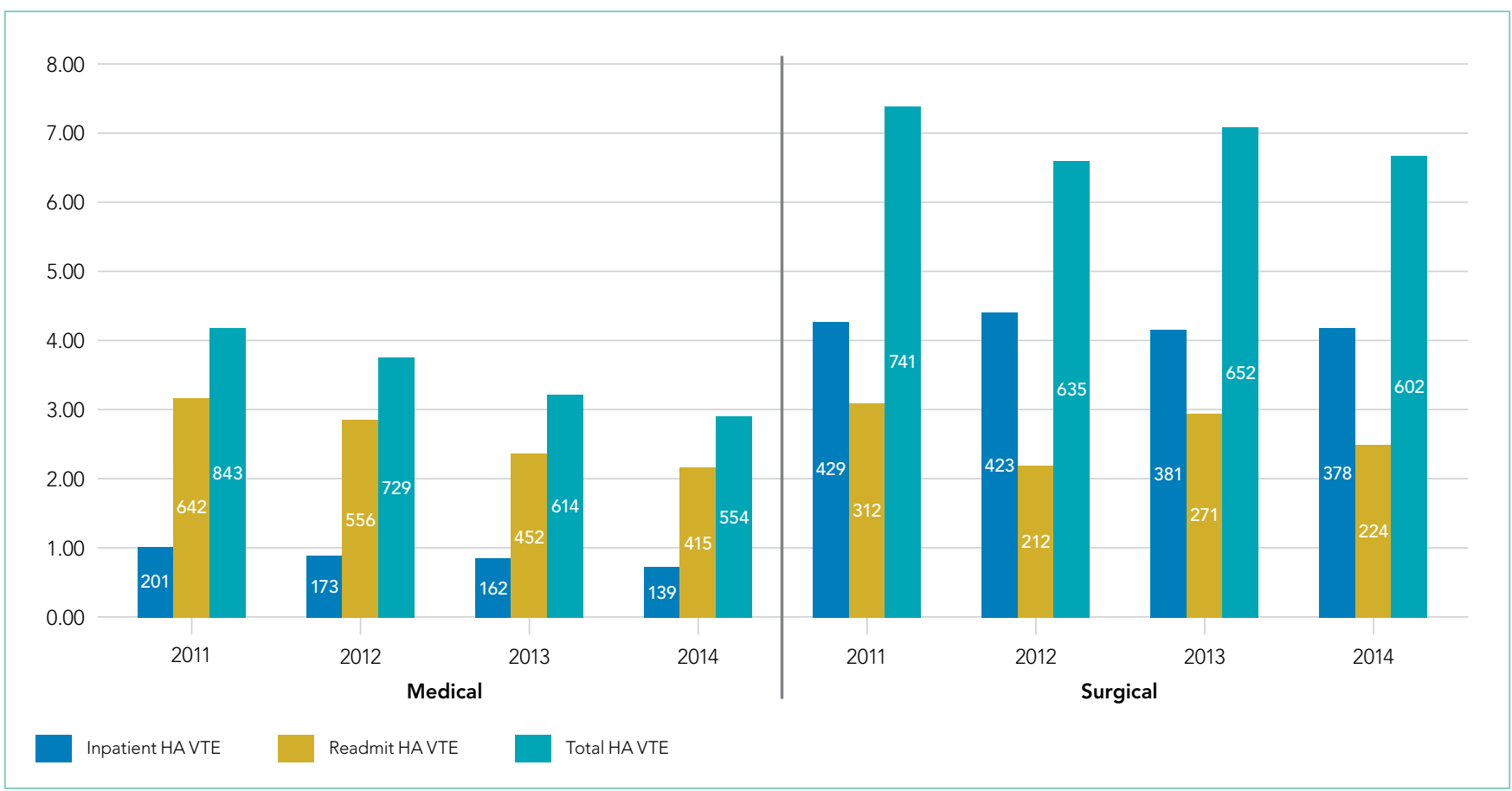

FIG 3. Medical versus surgical HA-VTE rates per 1000 admissions, all 35 sites (pilot and spread sites combined). Hospital-associated venous thromboembolism (HA-VTE) are broken out into inpatient HA-VTE (not present on admission) and Readmit HA-VTE (no VTE on index admission, but readmitted within 30 days with new VTE).

admission HA-VTEs were reduced by 315 (RR 0.72; $95 \% \mathrm{Cl}, 0.65$ 0.80 ), while the reduction in NPOA HA-VTEs was less robust (RR $0.88 ; 95 \% \mathrm{Cl}, 0.79-0.99)$. Pilot sites enjoyed a more robust reduction in HA-VTEs than spread sites ( $26 \%$ vs $20 \%$ ), largely because the pilot cohort enjoyed a 34\% reduction in NPOA HA-VTEs and a $20 \%$ reduction in Readmit HA-VTEs, while the spread cohort only achieved reductions in Readmit HA-VTEs.

In medical patients, 289 fewer HA-VTEs occurred in 2014 than in 2011 (RR 0.69; 95\% Cl, 0.62-0.77). There was a 27\% improvement in NPOA HA-VTEs and a $32 \%$ reduction in Readmit HA-VTEs. In surgical patients, 139 fewer HA-VTEs occurred in 2014 versus 2011, which just failed to reach statistical significance (RR 0.90; 95\% Cl, 0.81-1.01). Surgical NPOA HA-VTE stayed essentially unchanged, while Readmit HA-VTE declined from 312 to 224 (RR 0.80; 95\% Cl, 0.67-0.95).

\section{Safety}

Rates of HIT and adverse effects because of anticoagulants were low (Table). The rate of HIT declined from 178 events in 2011 to 109 in 2014 (RR 0.66; 95\% Cl, 0.52-0.84), and the RR of anticoagulant adverse events remained stable (RR 1.01; 95\% $\mathrm{Cl}, 0.87-1.15)$.

\section{DISCUSSION}

Our Ql project, based on a proven collaborative approach and mentorship, 18,22,24 order set redesign, and active surveillance, was associated with $26 \%$ less VTEs in the pilot cohort and $20 \%$ less VTEs in the spread cohort. These gains, down to a final rate of approximately 4 HA-VTEs per 1000 admissions, occurred despite a low baseline HA-VTE rate. Dignity Health achieved these improvements in 35 hospitals with varied sizes, settings, ordering systems, and teaching statuses, achieving what is to our knowledge the largest VTE Ql initiative yet reported.

Implementation experiences were not systematically recorded, and techniques were not compared with a control group. However, we believe that Dignity Health's organizational commitment to improvement and centralized support were crucial for success. In addition, the pilot sites received grant support from the GBMF for intensive quality mentoring, a strategy with demonstrated value. ${ }^{23}$ Mentors and team members noted that system-wide revision to the computerized physician order entry system was easiest to implement, while active surveillance represented the most labor-intensive intervention. Other experiences echoed lessons from previous VTE mentorship efforts. ${ }^{17,18}$

The selection of a VTE protocol conducive to implementation and provider use was a key strategy. The ideal approach to VTE risk assessment is not known, ${ }^{12,26}$ but guidelines either offer no specific guidance ${ }^{7}$ or would require implementation of 3 different systems per hospital. ${ }^{4,5}$ Several of these are point scoring systems, which may have lower clinician acceptance or require programming to improve real-world use ${ }^{18,26,27}$; the Padua score was derived from a patient population that differs significantly from those in the United States. ${ }^{12}$ Our study provides more practical experience with a "3-bucket" model, which has previously shown high interobserver reliability, good clinician acceptance, and meaningful reductions of VTE, including in American patient populations. ${ }^{18,22,24}$

The value of VTE prophylaxis is still disputed in many inpatient groups. The overall rate of HA-VTE is low, so the per-patient benefit of prophylaxis is low, and many patients may be 
TABLE. Rates of HA-VTE, HIT, and Adverse Anticoagulant Effect Events

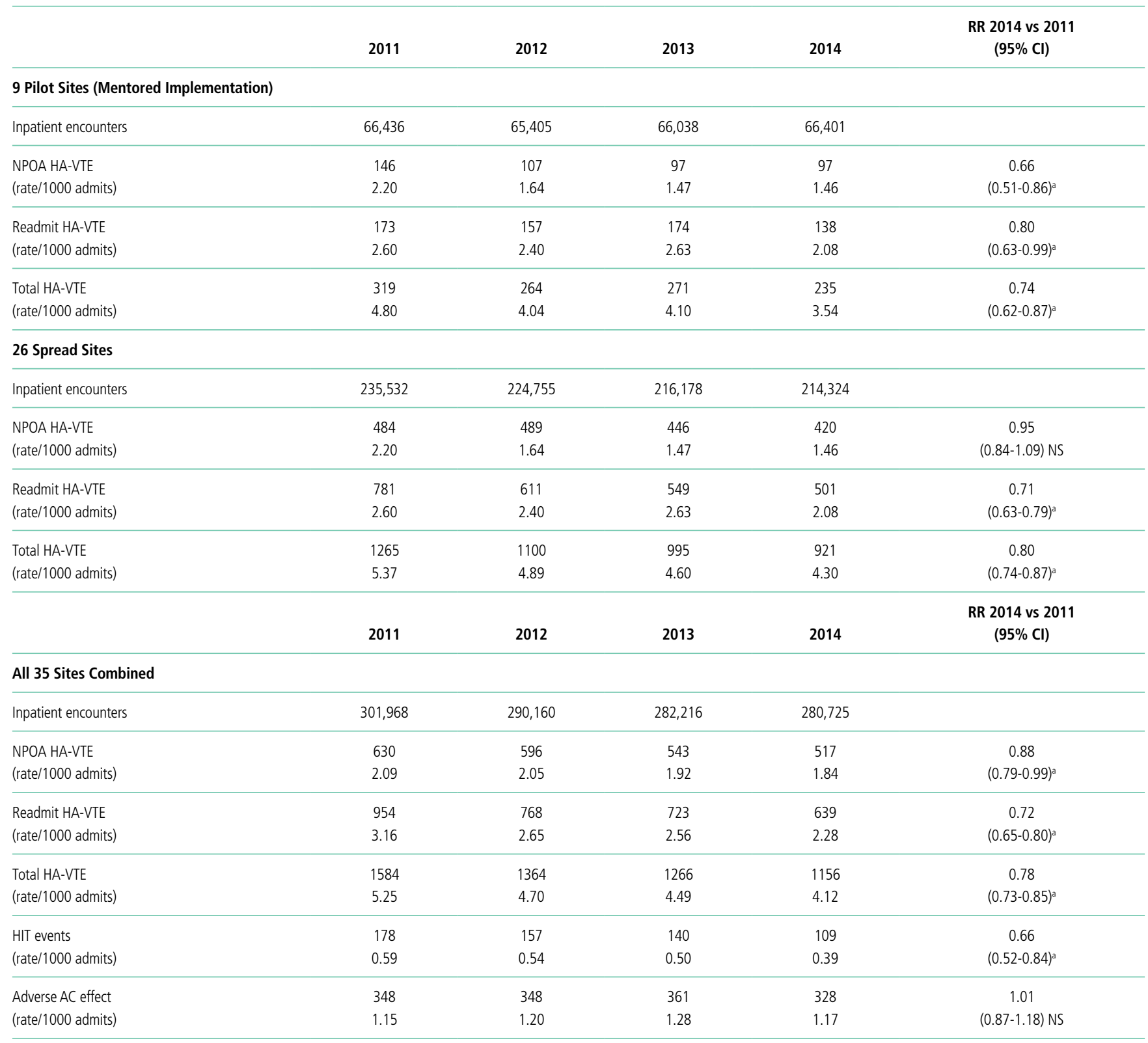

a Statistically significant.

NOTE: HIT and Adverse AC effect derived from administrative coding data and reflects impact of both therapeutic and prophylactic anticoagulant agents. Abbreviations: $\mathrm{AC}$, anticoagulant; $\mathrm{Cl}$ confidence interval; HA-VTE, hospital associated venous thromboembolism; HIT, heparin-induced thrombocytopenia; NPOA, not present on admission (acquired during the inpatient stay); NS, not statistically significant; RR, relative risk.

overprophylaxed. ${ }^{4,11,12}$ Recently, Flanders et al. ${ }^{20}$ reported that HA-VTE rates among 20,800 medical inpatients in Michigan were low (about 1\%) and similar at hospitals in the top (mean prophylaxis rate $86 \%$ ) or bottom (mean prophylaxis rate $56 \%$ ) tertiles of performance. Possible explanations for the differences between their multicenter experience and ours include our sample size (55 times larger) and the possibility that targeting prophylaxis to patients at highest need (captured in our protocol-compliant prophylaxis rates) matters more than prophylaxing a percent of the population.

Further research is needed to develop simple, easy-to-im- plement methods to identify inpatients who do not, or no longer, require prophylaxis. ${ }^{12}$ Hospital systems also need methods to determine if prophylaxis improvement efforts can lower their HA-VTE rates and in which subpopulations. For example, a collaborative effort at the University of California lowered HA-VTE rates toward a common improved rate of $0.65 \%$ to $0.73 \%$, ${ }^{22}$ while Dignity Health achieved improvement despite starting with an even lower baseline. In the University of California collaborative, benefits were limited chiefly to surgical patients, while Dignity Health achieved most improvement in medical patients, particularly in Readmit HA-VTE. If future re- 
search uncovers the reasons for these differences, it could help hospitals decide where to target improvement efforts.

Our study has several limitations. First, we used a nonrandomized time series design, so we cannot exclude other potential explanations for the change in VTE rates. However, there were no major changes in patient populations or concurrent projects likely to have influenced event rates. While we did not collect detailed demographic information on subjects, the broad inclusion criteria and multicenter design suggests a high degree of generalizability. Second, we followed inpatient VTE events and VTE-related readmissions, but not VTE treated in the outpatient setting. This did not change over the study, but the availability of all-oral therapy for VTE could have caused underdetection if clinic or emergency room doctors sent home more patients on oral therapy instead of readmitting them to the hospital. Third, implementation was enhanced by GBMF funds (at 9 sites, with the remainder benefitting from their experience), a shared electronic medical record at many sites, and a strong organizational safety culture, which may limit generalizability. However, spread sites showed similar improvement, paper-based sites were included, and the mentorship and quality collaborative models are scalable at low cost. Fourth, some Ql efforts began at some pilot sites in CY 2011, so we could not compare completely clean pre- and postproject timeframes. However, early improvement would have resulted in an underestimation of the project's impact. Lastly, the reason for a decline in HIT rates is not known. Standardized order sets promoted preferential use of low molecular weight heparin, which is less likely to induce HIT, and active surveillance targeted overprophylaxis as well as underprophylaxis, but we do not have data on heparin utilization patterns to confirm or refute these possibilities.

Strengths of our study include reductions in HA-VTE, both with and without access to GBMF funds, by using broadly available Ql strategies. ${ }^{17}$ This real-world success and ease of dissemination are particularly important because the clinical trials of prophylaxis have been criticized for using highly selected patient populations, ${ }^{11}$ and prophylaxis $\mathrm{Q}$ l studies show an inconsistent impact on VTE outcomes. ${ }^{15}$ In previous studies, two of the authors monitored orders for prophylaxis ${ }^{22,24}$; during this project, delivery for both pharmacologic and mechanical VTE prophylaxis was monitored, confirming that patient care actually changed.

\section{CONCLUSION}

Our multicenter VTE prophylaxis initiative, featuring a "3-bucket" VTE protocol, Ql mentorship, and active surveillance as key interventions, was associated with improved prophylaxis rates and a reduction in HA-VTE by $22 \%$ with no increase in adverse events. This project provides a model for hospital systems seeking to optimize their prophylaxis efforts, and it supports the use of collaborative $\mathrm{Ql}$ initiatives and SHM's quality mentorship program as methods to drive improvement across health systems.

Disclosure: None of the authors have any conflicts of interest related to any topics or products discussed in the article. Dignity Health provided a stipend for writing the manuscript to GM and IJ, as noted in the article, but had no role in data analysis, writing, or decision to submit.

\section{References}

1. U.S. Department of Health and Human Services; National Heart, Lung, and Blood Institute. Surgeon General's Call to Action to Prevent Deep Vein Thrombosis and Pulmonary Embolism. Rockville: Office of the Surgeon General; 2008.

2. Heit JA, Melton LJ, Lohse CM, et al. Incidence of venous thromboembolism in hospitalized patients versus community residents. Mayo Clin Proc. 2001;76(11):1102-1110.

3. Guyatt GH, Eikelboom JW, Gould MK. Approach to Outcome Measurement in the Prevention of Thrombosis in Surgical and Medical Patients: Antithrombotic Therapy and Prevention of Thrombosis, 9th ed: American College of Chest Physicians Evidence-Based Clinical Practice Guidelines. Chest. 2012;141(2 suppl):e185S-e194S. doi:10.1378/chest.11-2289.

4. Kahn SR, Lim W, Dunn AS, et al. Prevention of VTE in Nonsurgical Patients: Antithrombotic Therapy and Prevention of Thrombosis, 9th ed: American College of Chest Physicians Evidence-Based Clinical Practice Guidelines. Chest. 2012;141(2 suppl):e195S-e226S. doi:10.1378/chest.11-2296.

5. Gould MK, Garcia DA, Wren SM, et al. Prevention of VTE in Nonorthopedic Surgical Patients. Chest. 2012;141(2 suppl):e227S-e277S.

6. Falck-Ytter Y, Francis CW, Johanson NA, et al. Prevention of VTE in Orthopedic Surgery Patients: Antithrombotic Therapy and Prevention of Thrombosis, 9th ed: American College of Chest Physicians Evidence-Based Clinical Practice Guidelines. Chest. 2012;141(2 suppl):e278S-e325S. doi:10.1378/ chest.11-2404

7. Qaseem A, Chou R, Humphrey LL. Venous Thromboembolism Prophylaxis in Hospitalized Patients: A Clinical Practice Guideline from the American College of Physicians. Ann Intern Med. 2011;155(9):625-632.

8. The Joint Commission. Performance Measurement Initiatives. http://www. jointcommission.org/PerformanceMeasurement/PerformanceMeasurement. Accessed June 14, 2012.

9. National Quality Forum. National Voluntary Consensus Standards for Prevention and Care of Venous Thromboembolism: Policy, Preferred Practices, and Initial Performance Measures. http://www.qualityforum.org/Publications/2006/12/National_Voluntary_Consensus_Standards_for_Prevention_ and_Care_of_Venous_Thromboembolism__Policy,_Preferred_Practices,_ and_Initial_Performance_Measures.aspx. Accessed June 14, 2012.

10. Medicare Quality Improvement Committee. SCIP Project Information. Agency for Healthcare Research and Quality. http://www.qualitymeasures.ahrq. gov/content.aspx?id=35538\&search=scip. Accessed March 2013.

11. Lederle FA, Zylla D, MacDonald R, Wilt TJ. Venous Thromboembolism Prophylaxis in Hospitalized Medical Patients and Those with Stroke: A Background Review for an American College of Physicians Clinical Practice Guideline. Ann Intern Med. 2011;155(9):602-615.

12. Rothberg MB. Venous thromboembolism prophylaxis for medical patients: who needs it? JAMA Intern Med. 2014;174(10):1585-1586.

13. Cohen AT, Tapson VF, Bergmann JF, et al. Venous thromboembolism risk and prophylaxis in the acute hospital care setting (ENDORSE study): A multinational cross-sectional study. Lancet. 2008;371(9610):387-394.

14. Amin AN, Stemkowski S, Lin J, Yang G. Inpatient thromboprophylaxis use in U.S. hospitals: adherence to the seventh American College of Chest Physician's recommendations for at-risk medical and surgical patients. J Hosp Med. 2009;4(8):E15-E21.

15. Kahn SR, Morrison DR, Cohen JM, et al. Interventions for implementation of thromboprophylaxis in hospitalized medical and surgical patients at risk for venous thromboembolism. Cochrane Database Syst Rev. 2013;7:CD008201. doi:10.1002/14651858.CD008201.pub2.

16. Lau BD, Haut ER. Practices to prevent venous thromboembolism: a brief review. BMJ Qual Saf. 2014;23(3):187-195.

17. Maynard G. Preventing hospital-associated venous thromboembolism: a guide for effective quality improvement, 2nd ed. Rockville: Agency for Healthcare Research and Quality; 2015. https://www.ahrq.gov/sites/default/ files/publications/files/vteguide.pdf. Accessed October 29, 2017

18. Maynard G, Stein J. Designing and Implementing Effective VTE Prevention Protocols: Lessons from Collaboratives. J Thromb Thrombolysis. 2010;29(2):159-166.

19. Altom LK, Deierhoi RJ, Grams J, et al. Association between Surgical Care Improvement Program venous thromboembolism measures and postoperative events. Am J Surg. 2012;204(5):591-597.

20. Flanders SA, Greene MT, Grant P, et al. Hospital performance for pharmacologic venous thromboembolism prophylaxis and rate of venous thromboem- 
bolism: a cohort study. JAMA Intern Med. 2014;174(10):1577-1584.

21. Finn KM, Greenwald JL. Update in Hospital Medicine: Evidence You Should Know. J Hosp Med. 2015;10(12):817-826.

22. Jenkins $I H$, White $R H$, Amin $A N$, et al. Reducing the incidence of hospital-associated venous thromboembolism within a network of academic hospitals: Findings from five University of California medical centers. J Hosp Med. 2016;11(Suppl 2):S22-S28

23. Maynard GA, Budnitz TL, Nickel WK, et al. 2011 John M. Eisenberg Patient Safety and Quality Award. Mentored Implementation: Building Leaders and Achieving Results Through a Collaborative Improvement Model at the National Level. Jt Comm J Qual Patient Saf. 2012;38(7):301-310.

24. Maynard GA, Morris TA, Jenkins IH, et al. Optimizing prevention of hospi- tal-acquired venous thromboembolism (VTE): Prospective validation of a VTE risk assessment model. J Hosp Med. 2010;5(1):10-18.

25. The Joint Commission. Venous Thromboembolism Quality Measures. https://www.jointcommission.org/venous_thromboembolism/. Accessed October 13, 2017.

26. Maynard GA, Jenkins IH, Merli GJ. Venous thromboembolism prevention guidelines for medical inpatients: Mind the (implementation) Gap. J Hosp Med. 2013;8(10):582-588.

27. Elias P, Khanna R, Dudley A, et al. Automating Venous Thromboembolism Risk Calculation Using Electronic Health Record Data upon Hospital Admission: The Automated Padua Prediction Score. J Hosp Med. 2017;12(4): 231-237. 